\title{
Immortal time bias
}

\author{
Shengping Yang, PhD, Gilbert Berdine MD
}

I am planning a study to evaluate the relationship between infection and mechanical ventilation. Because we are interested in a time-to-event outcome, I am wondering whether there is a potential risk of introducing immortal time bias and how to avoid it.

In studies with a time-to-event (survival) outcome, immortal time refers to a period of follow-up during which, by design, death or the event of interest cannot occur. ${ }^{1,2}$ In general, immortal time could happen in an exposure/treatment group if the subjects need to be followed long enough to experience an exposure or receive a treatment and be event-free prior to the exposure/treatment.

\section{THE IMMORTAL tIME BIAS}

Figure 1 is a graphical illustration of immortal time. Each blue horizontal bar represents a subject, and the ones with an arrow on the right are those who have not experienced an event, while those with a vertical line are those who had experienced an event at the end of the follow-up. For the unexposed group, an event can occur any time after the start of the follow-up period. However, for the exposed group, an event cannot occur before the start of the exposure/treatment, otherwise, the subjects with an event prior to the exposure/treatment, by definition, are included in the unexposed group. In other words, the event-free time for the exposed group cannot be shorter than the interval between the start of the exposure and the start of follow-up. As a result, the average eventfree time for the exposed group is longer than that for the unexposed group, even when the exposure/ treatment has no effect.

Corresponding author: Shengping Yang Contact Information: Shengping.Yang@pbrc.edu DOI: 10.12746/swrccc.v10i42.991

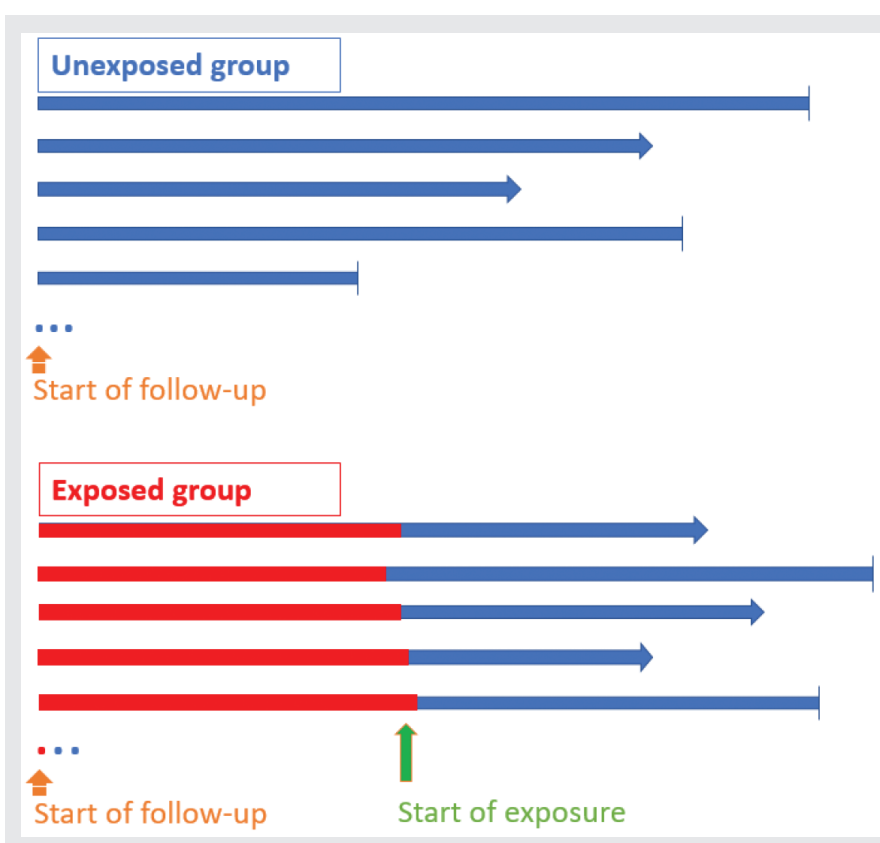

Figure 1. An illustration of immortal time. Each blue horizontal bar represents a subject, and the ones with an arrow (or a vertical line) on the right are those without (or with) an event at the end of follow-up. For the unexposed group, an event can occur any time after the start of follow-up (orange arrow); for the exposed group, an event cannot occur before the start of exposure (green arrow; differ slightly among individuals), and the horizontal bars in red represent the immortal time.

Since its first identification in the 1970s, immortal time bias has become a well-known bias and several studies have shown that results from observational studies can be invalid if immortal time bias is not accounted for. ${ }^{3,5-8}$

\section{THE DEMONSTRATION OF IMMORTAL TIME BIAS}

Dated back to 1885 , there were suggestions that Popes seem to live longer than artists, and an 
intuitive explanation is that an individual must survive long enough to become a Pope, compared to becoming an artist. ${ }^{4}$

In cohort studies as illustrated in Figure 1, the primary analysis is often to evaluate the association between the exposure/treatment and the occurrence of the event of interest. In general, survival data analysis methods, such as the Cox regression model, are commonly used.

When the determination of a subject's exposure/ treatment status involves a delay or wait period after the start of follow-up, immortal time bias arises. Here are two of the analytic methods that do not appropriately account for such a bias.

\subsection{GROUPING SUBJECTS BY FUTURE EXPOSURE/TREATMENT STATUS}

Specifically, subjects will be grouped by whether they experience an exposure during the follow-up period. Due to the observational nature of the study (Figure 1), subjects who did not live long enough to experience an exposure will be automatically included in the unexposed group. Therefore, the average event-free time will be artificially shortened for the unexposed group, compared to that for the exposed group. In other words, only those who live long enough are eligible to be included in the exposed group; this is analogous to the suggestion that Popes live longer than artists, because even if there were no exposure/treatment benefits, the exposed group would have longer average eventfree time.

It is worth noting that for subjects in the exposed group, prior to the exposure/treatment, their status was considered misclassified as "exposed", and this is an alternative interpretation of the bias mentioned above. Should the exposure/treatment occur at the start of follow-up without delay, such a bias would not exist, nor the immortal time.

Immortal time bias is a bias originated from study design; it cannot be completely removed by artificially excluding the period of immortality.

\subsection{EXCLUDE THE PERIOD OF IMMORTALITY}

While classifying subjects based on their future exposure introduces bias, excluding the period of immortality also introduces bias. Some studies simply redefine the start of follow-up as the start of the exposure. However, this cannot completely remove the immortality time bias, because, for example, if a disease has a higher early event rate, and if the period of immortality is excluded from the data analysis, then subjects in the unexposed group would be followed earlier than those in the exposed group. ${ }^{7}$ Consequently, the event rate in the unexposed group would be higher than that in the exposed group.

\section{Methods accounting for immortal time}

There are different analytical methods to account for immortal time.

\subsection{TIME-DEPENDENT EXPOSURE ANALYSIS}

Immortal time can be accounted for by introducing a time-dependent variable to define the exposure status of each subject. ${ }^{1,8}$ This is perhaps the most efficient way to account for immortal time. A timedependent variable is a predictor whose value is allowed to change over the follow-up period. For example, for subjects in the exposed group, the exposure status could be defined as "no" before experiencing the exposure, and then be changed to "exposed" after experiencing the exposure/treatment. For subjects in the unexposed group, the exposure status can always be "no" from the start to the end of the follow-up. In this way, the exposure status can be more accurately defined in data analysis. Note that this method can also accommodate situations in which the exposure status changes multiple times within a subject during the follow-up period. Many statistical software packages can be used for modeling time-dependent variables, including SAS, SPSS, Stata and R.

\subsection{Matching}

To implement matching, it is required that, at the design stage, an unexposed subject must be alive at 
the time at which the matching exposed subject is to experience the exposure. ${ }^{1,8}$ By doing this, the difference in follow-up time between the unexposed and the exposed group can be mitigated. Under matching, the start of the follow-up becomes the start of the exposure, and the time between subject accrual and exposure is balanced for the matched unexposed and exposed subjects. Note that the disadvantage of matching is that subjects who have experienced an event earlier are unlikely to be included in the analysis.

\subsection{LANDMARK APPROACH}

The exposure status of both the unexposed and exposed groups is determined at a certain prespecified point in time (landmark). ${ }^{1}$ This time will be the same for all subjects and be used as the start of the follow-up period. Like matching, many of the subjects might not be included in the data analysis, depending on the pre-specified time. On the other hand, if the pre-specified time is far ahead of the average exposure/ treatment starting time, then the immortal time bias might not be fully accounted for.

\section{Other considerations}

The immortal time bias does not apply to an intention-to-treat analysis in a randomized controlled trial. ${ }^{1}$ In a randomized trial, although it is possible that the exposure/treatment starts sometime after baseline, subjects who are randomly assigned to the exposure/treatment group will be treated as "exposed" regardless of whether they have experienced an event prior to the planned exposure/treatment. Sometimes the exposure/treatment status of certain subjects can change during a trial; however, these changes will be ignored in data analysis to preserve the benefit of randomization and to provide an unbiased estimate of the efficacy of the intervention at the level of adherence in the study.

While it is well known that bias can arise due to confounding in an observational study, the magnitude of immortal time bias can be greater. For example, if the duration of the immortal time is large, then it could lead to greater bias in the analysis. In addition, unlike confounding, immortal time bias often results in inflated exposure/treatment effect, and these positive results are easier to publish. Immortal time bias is particularly important for studies evaluating COVID vaccines as the vaccination process is not instantaneous, but takes weeks to complete. For some vaccines, two separate injections separated by 14 days are required, and there is also the uncertain period for 14 days following the second injection. Boosters have further complicated the immortal time bias of COVID vaccination status. During the vaccination process, the status of the individual is neither unvaccinated nor fully vaccinated, so the analysis can be quite different if these patients are included with the vaccinated or the unvaccinated.

In conclusion, in observational studies, immortal time bias arises when there is a delay or wait period before a subject's exposure/treatment status can be determined. To avoid such a bias, the exposure/treatment should start at the beginning of the follow-up. Should a delay or period of immortality be not avoidable, analytical methods are available to account for such a bias, including using a time-dependent exposure analysis, matching, and the landmark approach. Note that although these methods can account for immortal time bias, there might be other limitations.

Keywords: immortal time bias, observational studies, events, outcomes

Article citation: The Southwest Respiratory and Critical Care Chronicles 2022;10(42):47-50

From: Department of Biostatistics (SY), Pennington Biomedical Research Center, Baton Rouge, LA; Department of Internal Medicine (GB), Texas Tech University Health Sciences Center, Lubbock, Texas

Submitted: $1 / 7 / 2022$

Accepted: 1/10/2022

Conflicts of interest: none

This work is licensed under a Creative Commons Attribution-ShareAlike 4.0 International License. 


\section{REFERENCES}

1. Dekkers OM, Groenwold RHH. When observational studies can give wrong answers: the potential of immortal time bias. Eur J Endocrinol 2021;184(1):E1-E4.

2. Lee H, Nunan D. Immortal time bias. In: Catalogue of Bias. 2020 https:/catalogofbias.org/biases/immortaltimebias/

3. Lévesque LE, Hanley JA, Kezouh A, et al. Problem of immortal time bias in cohort studies: Example using statins for preventing progression of diabetes. BMJ 2010;340:907-11.

4. Shariff SZ, Cuerden MS, Jain AK, et al. The secret of immortal time bias in epidemiologic studies. J Am Soc Nephrol 2008;19:841-843.
5. Suissa S. Effectiveness of inhaled corticosteroids in chronic obstructive pulmonary disease: Immortal time bias in observational studies. Am J Respir Crit Care Med 2003;168: 49-53.

6. Suissa S. Immortal time bias in observational studies of drug effects. Pharmacoepidemiol Drug Saf 2007;16:241-9.

7. Suissa S. Immortal time bias in pharmacoepidemiology. Am J Epidemiol 2008;167:492-499.

8. Zhou Z, Rahme E, Abrahamowicz M, et al. Survival bias associated with time-to-treatment initiation in drug effectiveness evaluation: A comparison of methods. Am J Epidemiol 2005;162:1016-23. 